\title{
LEGIBILITY
}

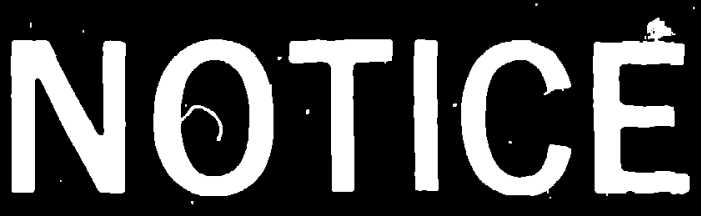

A major purpose of the Technical Information Center is to provide the broadest dissemination possible of information contained in DOE's Research and Development Reports to business, industry, the academic community, and federal, state and local governments.

Although a small portion of this report is not reproducible, it is being made available to expedite the availability of information on the research discussed herein. 
LA-UR $-89-2148$

\title{
LA-CR- - 89-2148
}

\section{DE89 014289}

TITLE DETECTION OF PHOTON BURSTS FROM SINCILE 200 OV Mg IUNS, I'ROCRF.SS IN PHOTON BLIRST MASS SPECTROMETRY

AUTHOA(S) W. M. Falrbank, Jr., R. D. Labelle, R. A. Keller, and E. P. Chamberlin

SUbmitted to Proceedings of the 9 th International Conference on Laser Spectroscopy. June 19-23, 1989, New Hampshire

\section{DISCY.AIMER}

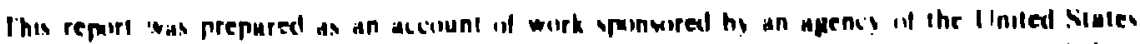

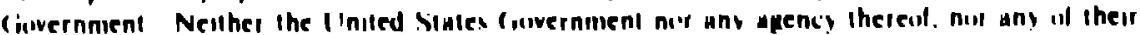

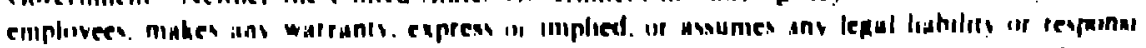

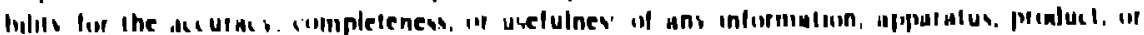

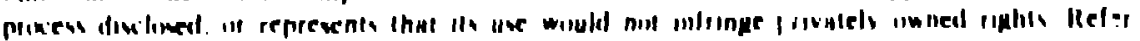

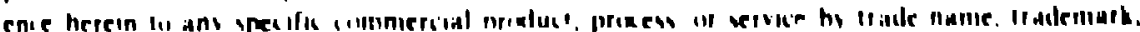

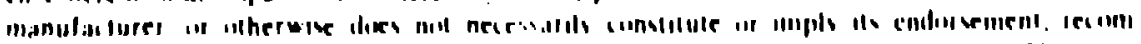

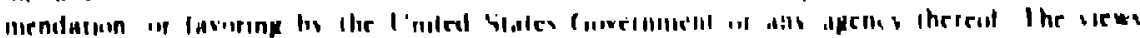

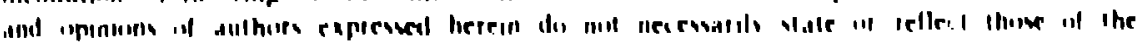

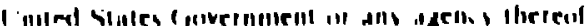

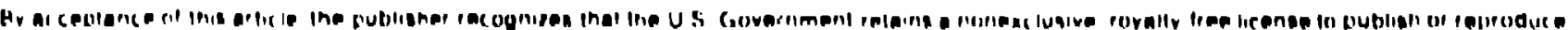


Detection of Photon Buists from Single $200 \mathrm{eV}$ Mg Ions, Progress in photon Burst Mass spectrometry

W. M. Faizbank, Iz. and R. D. Laselle

Department oz Physics, Colozado State Universizy

Fort Collins, CO 80523, USA

R:chard. A. Keller and E. Philip Chamberlin

Los Alamos National Laborazory,

Los Alamos, NM 87545, USA

Laser Spectroscopy IX, Springer-Verlag, 1939, in press.

Modern atom counting methods, based on adyances in laser and acceleratoz technology, provide a valuable complement to traditional decay counting methods for radioisorope dating and tracer work. Tandem Accelerator Mass Spectrometry (TAMS) has already had a large impact on ${ }^{4} \mathrm{C}$ dating and is beginning to provide new opportunities with ${ }^{10} \mathrm{Be}$ and several other isotopes. We report here on progress in the development of a laser-based technique, photon Burst Mass Spectrometry, $\therefore 2$ which is potentially capable of analyzing many of the elements which are forbidden in TAMs because they do not fcrm negative ions. We are especially interested in. the noble gases, which have a variety of potential scientific and environmental appilications. ${ }^{3}$

Photon Burst Mass Spectrometry (PBMS) is based on the coupling of a highly selective laser single atom or ion counter, the photon burst detector, with a mass spectrometer, which serves as an on-line prefilter to reduce the abundant isotopes by $10^{6}$ (EIg. 1). In two previous papers, calculations have been presented for several sample isotopes, which indicate that isotope ratios as low as $10^{-15}$ are measurable in 30 hours or less by the PaMs method. ${ }^{2.4}$ In the last year or so, our primary effort has been in demonstrating experimentally the parameters lipon which these calculations were hased and in designing and testing the Important components of the proposed PBMS instrument. Progress on these go.ris is the mafor focus of this paper.

The piroton burst detector is being developed and tested with a siniple Colutzon lon source, equipped with an ExB (Wien) filter. It provides mass-selected $\mathrm{Mg}^{\circ}$ ions at $200 \mathrm{eV}$ at a low current on the order of picoamps. The lons pass

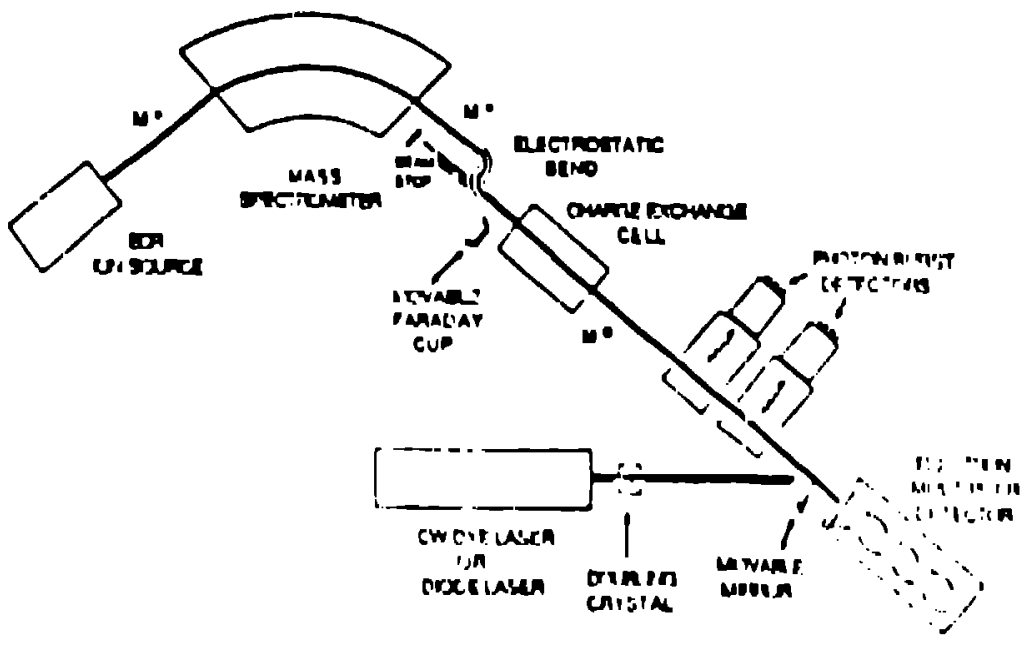

Fig. ?. Photon Burst Ma:ss :pprearcimpting. 
collinearly through a photon burst detector with a focussed laser beam tuned to the ${ }^{2} \mathrm{~S}_{1 / 2}-{ }^{2} \mathrm{P}_{3 / 2} \mathrm{Mg}^{+}$resonance at $279.5 \mathrm{~nm}$. Focussing was required because the laser power, $: 0 \mu \mathrm{w}$, was insufficient to saturate the full diameter of the ion beam.

Results obtained with the mass filter of are shown in Fig. 2. In the total fluorescence signal, peaks are seen from all three isotopes, but a large stray light background $\left(4 \mathrm{x} 10^{-9}\right.$ of the incident light) limits the signal-tobackground ratio to abcut 2. Much greatez contrast is seen in the burst signal, with nearly zero background. The steepness of the sides of the peaks indicates that the contributions from neighboring isotopes at line center are well below the stray light background. With measured experimental parameters and an estimated 7 के collection/quantum efficiency, predicted maximum values for the two ${ }^{25} \mathrm{Mg}^{+}$and ${ }^{26} \mathrm{Mg}^{2}$ peaks from jeft to right are 83,1500 and 6450 bursts/sec, respectively. The predicted stray light background is 2.2 bursts/sec, including measured effects of double pulsing in the photomultiplier. All these predictions agree well (within a factor of two or so) with the observations. The derived average burst size for resonant ${ }^{25} \mathrm{Mg}+$ and ${ }^{26} \mathrm{Mg}^{+}$lons is, respectively 3.1 and 4.5 counts.

This is the first time that photon bursts from single ions, other than in a trap, have been observed. It is also the first time that superthermal particles have been detected by the photon burst method. It is worth noting that the high optical efficiency and burst sizes demonstrated in these experiments, as well as the good agreement of the experimental data with theoretical predictions, represent impoztant milestones in the development and testing of the PBMS method. The relatively high stray light level in these experinients, due partly to light from the lon source, prevented a better demonstration of the isotoplc selectivity of the photon burst method. Nevertheless, an abundance sensitivity of at lease $10^{3}$ is shown in E1g. 2 . The calculated average background from nelghboring isotopes is $10^{4}$ lower than the stray light level, and contributed negligibly to background bursts.

E Ig. 2. I luorescence and photon burgt spectra of M.g long obtalned with the wien fllter off: (a) total count rate, and (b) rate of bursts with $\geq 5$ counts. Cur's $(a)$ represents the calculatel burst rate 1 t the photon arcival rate $\ln$ (a) wire? uncorrelatod, excopt. for a 0.3 afterpuleing correction. from the left the prak:; ir!: $\therefore \mathrm{Mg}^{\circ}, " \mathrm{Mg} \mathrm{H}^{\circ}\left(\mathrm{E}^{\prime}-\mathrm{H}-\mathrm{E}^{\prime}-1,2,3\right)$, $\therefore M 0^{\circ}\left(F^{\prime \prime}=3-F^{\prime}-2.3 .4\right)$. 1nd
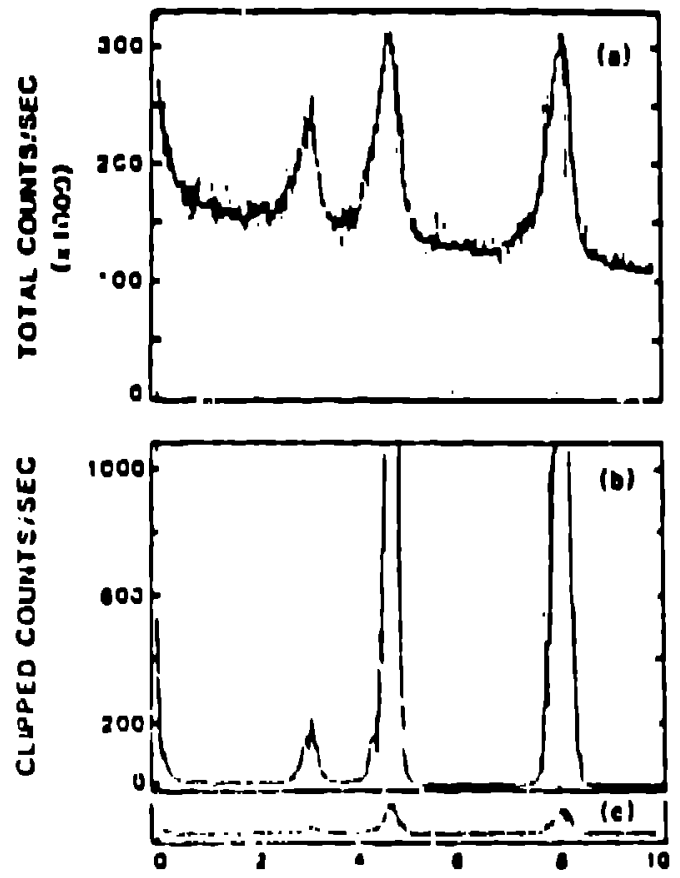
Recentiy we have installed an electrostatic bend between the ion source and the pnoton burst detector, as illustrated in the FBMS diagram (Fig. 1). This efEectively eliminates background photons from ion source. A stray light level of $10^{-10}$ for the whole system has been achieved at $280 \mathrm{~nm}$. In more careful work with the photon burst detector alone, $10^{-11}$ was measured at $280 \mathrm{~nm}$ and $-\mathrm{J}^{-: 2}$ with a clean TEM 00 beam at 633 $\mathrm{nm}$. It is anticipated th. proper spatial filtering of the beam with a pinhole or single-mode optical fiber will allow the latter level to be reached at all wavelengths. Thus we have demonstrated essentially all of the required features of the proposed photon burst detector.

The planned mass spectrometer for our full PBMS demonstration is a $126^{\circ}$ magnesic isotope separator at Los Alamos National Laboratory. ${ }^{5}$ A microwave source for this instrument ${ }^{6}$ has been installed and tested. It produces a $10 \mu A$ current in a $1-2 \mathrm{~mm}$ spot at the exit of the spectrometer with $50 \%$ overall ionization and transmission efficiency and reasonable divergence. An abundance sensitivity of $10^{6}$ at the krypton masses has been measured. The background isobars observed at the $10^{-6}$ level pose no problem due to the high isobaric selectivity of the photon burst detector. Thus most of our assumed mass spectrometer specification are exceeded by this instrument.

Many of the important features of the Photon Burst Mass Spectrometry method, including selective counting of single fast ions, have now been confirmed experimentally. Progress is being made toward adapting an isotope separator at Los Alamos National Laboratory for the Inal demonstration of the full capabilities of the PBMS method. Further studies are stili required on the most important remaining uncertainty in the method, the magnitude of longitudinal veloclty changes during charge exchange to metastable atomic states. This is not thougit to be a severe problem because most charge exchange collisions occur mainly at large impact parameters.

\section{References}

1.R. A. Keller, D. S. Bomse and D. A. Cremers, Laser Focus (October 1981) p.75.

2. W. M. Fairbank, Jr., Nucl. Instr. and Methods B29, 407 (1987).

3.B. E. Lehmann and H. H. Loosl1, "Use of noble gas radiolsotopes for environmental research", in Besonance Iontratton spectroscapy 1984, G. S. Hurst and M. G. Payne, eds. (Institute of Physics, Bristol, 1984) p.219.

4. W. M. Fairbank, Jr, et al., "Prospects for large dynamic range lsotope analysis using phuton burst mass

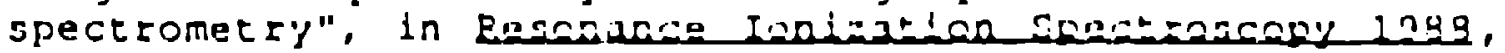
'I. Lucatorto and $J$. E. Pazks, eds. (Institut! of Physics, $1989) \mathrm{p}, 53$.

5. E. P. Chamberlin et al., Mugl. Instr. and Mothods L25, 21. (1987). 